\title{
Increase of sodium current after pyrethroid insecticides in mouse neuroblastoma cells
}

\author{
Gé S.F. Ruigt*, Hans C. Neyt, Johan M. Van der Zalm** and Joep Van den Bercken \\ Department of Veterinary Pharmaculogy, Pharmacy and Toxicology, University of Utrecht, Utrecht (The Netherlands)
}

(Accepted 16 June 1987)

Key words: Pyrethroid; Neurotoxin; Sodium channel gating; Neuroblastoma; Temperature

\begin{abstract}
The effects of 4 different pyrethroid insecticides on sodium channei gating in internally perfused, cultured mouse neuroblastoma cells (N1E-115) were studied using the suction pipette, voltage ciamp technique. Pyrethroids increased the amplitude of the sodium current, sometimes by more than $200 \%$. Activation of the sodium current occurred at more hyperpolarized potentials than under control conditions. The declining phase of the sodium current during depclarization was markedly slowed down and after repolarization of the membrane a large, slowly decaying sodium tail current developed. Pyrethroids did not affect the sodium current reversal potential, steady-state sodium inactivation or recovery from sodium channel inactivation. The amplitude of the pyrethroid-induced slow tail current was always proportional to the sodium current at the end of the preceding depolarizing pulse. The rate of decay of the slow tail current strongly depended on pyrethroid structure and increased in the order deltamethrin, cyphenothrin, fenfluthrin and phenothrin. The rate of decay further depended on membrane potential and temperature. Below $-85 \mathrm{mV}$ the instantaneous current-voltage relationship of the slow tail current showed a negative slope conductance. The tail current decayed more slowly at low temperatures. Arrhenius plots indicated that the relaxation of open sodium channels to a closed state involved a higher energy barrier for pyrethroid-affected than for normal channels. The energy barrier was higher after deltamethrin than after the non-cyano pyrethroid fenfluthrin. It is concluded that in mamınalian neuronal membrane pyrethroids selectively reduce the rate of closing of sodium channels both during depolarization and after repclarization of the nerve membrane.
\end{abstract}

\section{INTRODUCTION}

Pyrethroids constitute a large group of neurotoxic insecticides, which were originally developed as synthetic analogs of the natural pyrethrins present in the flower heads of certain Chrysanthemum species. There is ample evidence suggesting that pyrethroids primarily affect sodium channels in excitable membranes causing a prolongation of the sodium current during excitation. The prolonged sodium current results in the development of a depolarizing afterpotential following the action potential and is also responsible for the induction of repetitive activity which is the most characteristic effect of pyrethroid poisoning in the nervous system ${ }^{25.26 .31 .35 .38}$. The modification of sodium channel gating by pyrethroids has been studied in voltage clamp experiments on crayfish $^{18-20}$ and squid ${ }^{17}$ axon membranes, as well as in myelinated nerve fibers of the clawed frog ${ }^{36,37}$. These studies have revealed that the prolongation of the sodium current is mainly due to a reduced rate of closing of a fraction of sodium channels which are affected by pyrethroids in their cpen configuration. Lund and Narahashi ${ }^{18}$ further showed that the opening of a number of pyrethroid-affected sodium channels in squid and crayfish axons was slowed down.

Over the past few years the mouse neuroblastoma cell line, N1E-115, has proved to be a suitable model system for the study of ionic currents in mammalian neuronal membrane ${ }^{34}$. The characteristics of sodium, potassium and caicium currents are essentially similar to those observed in other excitable tis-

\footnotetext{
* Present address: Organon International SDG. RE2134. POB 20. 5340 BH Oss. The Netherlands.

** Present address: Duphar. Weesp. The Netherlands.

Correspondence: J. Van den Bercken. Department of Veterinary Pharmacology. Pharmacy and Toxicology. University of Utrecht. P.O. Box 80176. 3508 TD Utrecht. The Netherlands.
} 
sues $^{2 i-23}$. It has recently been shown by single channel analysis that sodium channel gating kinetics in neuroblastoma celis ${ }^{1.14}$ may deviate from the classical model of sodium channel gating ${ }^{10}$. Radioligand binding studies have provided much information on the interaction of a variety of neurotoxins with the sodium channels of culturer mouse neurobiastoma cells. Sodium channel neuroroxins have been divided into different groups which interact with different binding sites on the channel protein ${ }^{4.15}$. Detailed electrophysiological studies in neuroblastoma cells have revealed that toxins belonging to these bi )chemically defined groups also affect sodium channei gating in different ways ${ }^{8.9111 .29}$.

Although much is known about the action of pyrethroids on sodium currents in invertebrate and frog neuronal membranes, the action of these broadly used insecticides on mammalian sodium currents has not been investigated in detail. In neuroblastoma cells the pyrethroids deltamethrin ${ }^{12}$, kadethrin ${ }^{12}$, tralomethrin $^{30}$ and tralocythrin ${ }^{30}$ do not stimulate the entry of ${ }^{22} \mathrm{Na}^{+}$in neuroblastoma cells by themselves, but increase ${ }^{22} \mathrm{Na}^{+}$entry when applied in combination with toxins that keep the sodium channel open. This was also found for the insecticidally active enantiomers of deltamethrin and des-cyano deliamethrin in mouse synaptosomes ${ }^{6}$. Deltamethrin and kadethrin further induced a depolarizing afterpotential in neuroblastoma cells ${ }^{12}$. Other pyrethroids, like bioresmethrin, cis-permethrin and cismethrin were unable to stimulate ${ }^{22} \mathrm{Na}^{+}$entry and failed to affect the action potential. The inactive pyrethroids antagonized the effects of the active pyrethroids. It was concluded that the binding component for pyrethroids in neuroblastoma cells is distinct from that of other toxins known to affect the sodium channel. The pyrethroid tetramethrin has further been shown to increase the mean open time of single sodium channels in mouse neuroblastoma cells ${ }^{39}$.

The objective of the present study was to investigate whether pyrethroids have a similar effect on sodium currents in mammalian neuronal membrane as described for frog and invertebrate axonal membrane. Most experiments were performed with the active isomer of a pyrethroid with a pentafluorbenzyl alcohol moiety (fenfluthrin), which is a potent, rapidly acting insecticide ${ }^{27}$. This pyrethroid induces pronounced repetitive activity in the crayfish stretch re- ceptor organ $^{33}$ and in muscle fibers of the clawed frog $^{32}$. In the isolated frog node of Ranvier fenfluthrin induces a prolongation of the sodium current (unpublished) similar to that observed after other pyrethroids ${ }^{37}$.

\section{MATERIALS AND METHODS}

\section{Cell culture}

Experiments were performed using the mouse neuroblastoma cell line N1E-115 (ref. 2). Cells were routinely grown in Dulbecco's modified Eagle's medium (DMEM; Flow Laboratories), buffered with 45 $\mathrm{mM} \mathrm{NaHCO}$ and supplemented with $7.5 \%$ fetal calf serum (FCS, Flow Laboratories). Cultures were maintained at $37{ }^{\circ} \mathrm{C}$ in a humidified atmosphere containing $7.5 \% \mathrm{CO}_{2}$. Cells were subcultured every 5 days by washing with phosphate buffered saline (PBS) followed by a 5-min incubation period at $37^{\circ} \mathrm{C}$ in PBS containing 5\% trypsin. Trypsinized cells were resuspended in DMEM with $1.5 \%$ FCS and plated on tissue culture dishes. Two days after subculture $1 \%$ dimethyl sulfoxide (DMSO) was added. Dishes were regularly refreshed with new medium. Treatment with DMSO for 3 days up to 3 weeks inhibited cell division and initiated cell differentiation in most of the cells as judged by the outgrowth of neurites and the increase in cell size up to a final diameter of 50-100 $\mu \mathrm{m}$. The presence of long processes in morphologically differentiated cells generally made them unsuitable for voltage clamp experiments by means of the suction pipette technique, as it hindered an adequate space clamping of the membrane potential. Therefore most experiments were performed on isolated round cells.

\section{Experimental procedures}

A culture dish containing differentiated neuroblastoma cells was placed on an aluminum plate which was in direct contact with a Peltier element for temperature control. This assembly was mounted on the stage of an inverted phase-contrast microscepe. The temperature of the bathing solution was held constant at $18 \pm 0.1^{\circ} \mathrm{C}$. A suction pipette technique for combined voltage clamp and internal perfusion of neuroblastoma cells, modified after the method developed by Lee et al. ${ }^{16}$ was used. Suction pipettes were pulled in two stages and the tip was fire-po- 
lished under visual control until the final internal tip diameter was about $10 \mu \mathrm{m}$ resulting in a tip resistance of $400-600 \mathrm{k} \Omega$. Membrane potential was recorded by means of an $\mathrm{Ag} / \mathrm{AgCl}$ electrode which was connected through a $150 \mathrm{mM} \mathrm{KCl}$ agar bridge to the electrode holder. Current was passed through a piece of stainless steel tubing, which was coated with a layer of plastic except for the platinized tip. The tubing was advanced to the tip of the suction pipette and was simultaneously used for delivery of the internal perfusate. The fluid pressure at the pipette tip could be regulated accurately by applying negative pressure to the electrode holder. Before touching a cell with the pipette, pressure was adjusted to assure a small outflow of solution. The resistance between the suction electrode and the bath solution, which was constantly monitored by applying $15 \mathrm{~ms}$ constant hyperpolarizing current pulses of $1 \mathrm{nA}$, rose rapidly when the negative pressure was increased. Final seal resistance usually varied between 50 and $100 \mathrm{M} \Omega$ and often reached values of a few hundred MS. After breaking the aspirated membrane by means of a pressure jump or by preamplifier oscillation the response to the stimulus was altered due to the charging of the membrane capacitance. Thereafter suction was maintained at a negative pressure of 25-50 mbar.

\section{Voltage clamp and current measurements}

A conventional voltage clamp circuit was used with a capacity compensated high impedance preamplifier for recording of the membrane potential. The bathing solution was clamped at ground potential by means of a virtual ground circuit employing an $\mathrm{Ag} / \mathrm{AgCl}$ reference electrode and a platinum black current passing electrode. Current was measured by means of a current to voltage converter connected to the platinum black electrode. A fraction of the current was subtracted from the input signal to the voltage clamp control amplifier for series resistance compensation. Series resistance was estimated from the instantaneous voltage displacement produced by a current step under current clamp conditions. Its value ranged from 200 to $800 \mathrm{k} \Omega$ and was compensated for $80-95 \%$. The membrane potential was held at $-95 \mathrm{mV}$ and the holding current remained stable $(0.1 \pm 0.1 \mathrm{nA})$ for several hours. Stable recordings could be obtained for up to $8 \mathrm{~h}$. In order to remove resting sodium channel inactivation, depolarizing pulses were preceded by a conditioning hyperpolarization of $200 \mathrm{~ms}$ to $-125 \mathrm{mV}$. Pulse sequences were generated by a programmable, microprocessor-controlled square wave generator. To increase the effective dynamic range of the $A$ to $D$ converter, linear capacitative and leakage currents were subtracted electronically. Currents were digitized by means of an 8 bits transient recorder. The remainder of the linear capacitative and leakage current components was subtracted by using a $P / 2$ protocol ${ }^{28}$ in which the depolarizing pulse was preceded by two hyperpolarizing pulses of half-amplitude. Signals were averaged on-line with an HP-1000 minicomputer system and were stored on magnetic disc together with pulse information from the square wave generator. Voltage clamp currents were digitally filtered off-line before further analysis. Time constants were fitted by using a derivative-free Levenberg-Marquardt routine ${ }^{3}$.

\section{Solutions and chemicals}

Low sodium ion concentrations were used in external solutions in order to minimize non-uniform spaceclamp. Complete replacement of potassiuin by cesium internally as well as addition of tetraethylammonium chloride (TEACl) to the external solution adequately eliminated voltage-dependent potassium currents. The external solution contained (in $\mathrm{mM}$ ): $\mathrm{NaCl} 60, \mathrm{HEPES} 25, \mathrm{TEACl} 25, \mathrm{MgCl}_{2} \mathbf{0 . 8}, \mathrm{CaCl}_{2} 2$, glucose 30 and TMACl 60 (osmolarity $300 \mathrm{mOsm} / \mathrm{lit}$ er); $\mathrm{pH}$ was adjusted to 7.4 with TMAOH. The internal perfusate contained (in mM): Na-HEPIES 20, and $\mathrm{CsOH} 120 ; \mathrm{pH}$ was adjusted to 7.1 with L-glutamic acid and the osmolarity was adjusted to $310 \mathrm{mOsm} /$ liter with sucrose. The voltage-dependent sodium current could be readily blocked by $0.5 \mu \mathrm{M}$ TTX, even in the presence of pyrethroids. The following pyrethroids were used: pentafluorbenzyl-(1R,cis)-3(2,2-dichlorovinyl)-2,2-dimethyl-cyclopropane-carboxylate (fenfluthrin); 3-phenoxybenzyl-(1R,cis)-3(isobutenyl)-2,2-dimethyl-cyclopropane-carboxylate (phenothrin); $\alpha(\mathrm{S})$-cyano-3-phenoxybenzyl-(1R.cis)3-(isobutenyl)-2,2-dimethyl-cyclopropane-carboxylate (cyphenothrin) and $\alpha(\mathrm{S})$-cyano-3-phenoxybenzyl-(1R.cis)-3-(2,2-dibromovinyl)-2,2-dimethyl-cyclopropane-carboxylate (deltamethrin). The compounds were dissolved in DMSO and stored at $5^{\circ} \mathrm{C}$. Pyrethroids were either directly added to the culture 
dish or were prediluted in the superfusing saline. The final concentration of DMSO was always less than $0.1 \%$, which had no effect on the sodium current in control experiments.

\section{RESULTS}

\section{Membrane sodium currents}

Control sodium currents in response to a series of increasing depolarizing pulses are shown in Fig. 1A. Inward currents were characterized by rapid activation and inactivation kinetics. Treatment with fenfluthrin $(1 \mu \mathrm{M})$ affected both the amplitude and the time course of the sodium current (Fig. 1B). Three main effects of the pyrethroid were found: (1) the peak amplitude of the sodium current increased; (2) the declining phase of the sodium current during de-

A

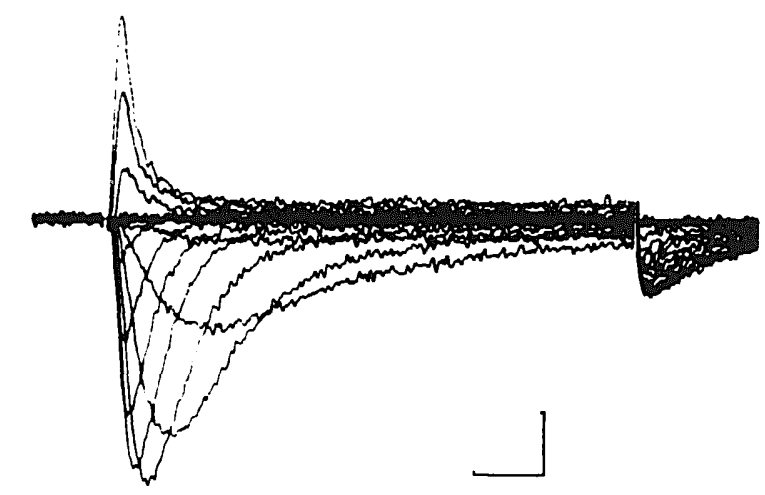

C

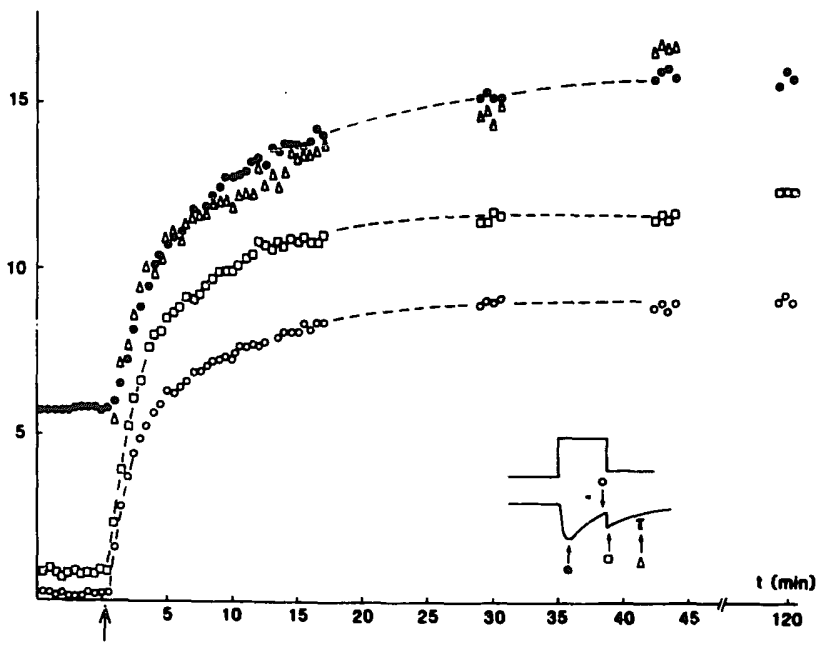

polarization was greatly slowed down resulting in a marked residual current towards the end of the $15 \mathrm{~ms}$ pulse; and (3) after repolarization of the membrane to the holding potential a large, slowly decaying sodium tail current remained.

The effects on the sodium current developed rapidly after addition of the pyrethroid to the external solution. Fig. 1C shows that after application of $1 \mu \mathrm{M}$ fenfluthrin the peak sodium current, the slow sodium tail current and the residual current increased in parallel. The rapid increase during the first 5-10 $\mathrm{min}$ of exposure was followed by a more gradual increase to a steady-state level which was reached after 45-60 min of exposure. Fig. 1C also shows that the time constant of the decay of the slow sodium tail increased with a similar time course. After higher concentrations of pyrethroid these effects developed fas-

B

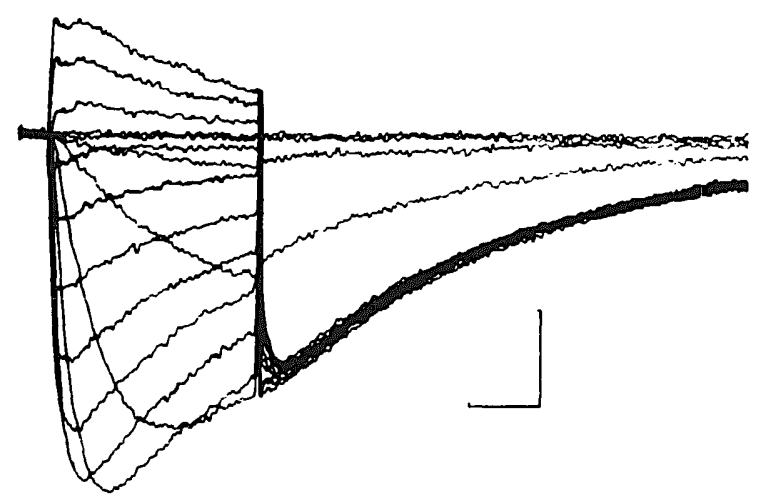

Fig. 1. A: control sodium currents in an internally perfused neuroblastoma cell evoked by a series of $15 \mathrm{~ms}$ depolarizing pulses with increasing amplitude. Pulse potentials increase from -65 to $75 \mathrm{mV}$ with an increment of $10 \mathrm{mV}$. Before each depolarization the membrane was conditioned at $-135 \mathrm{mV}$ for $200 \mathrm{~ms}$. Holding potential was $-95 \mathrm{mV}$. B: same as in A after treatment of the same cell with $1 \mu \mathrm{M}$ fenfiuthrin for $85 \mathrm{~min}$. C: development of the effect of $1 \mu \mathrm{M}$ fenfluthrin applied at $\uparrow$ on several parameters of the sodium current evoked by a $15 \mathrm{~ms}$ depolarizing pulse to $-5 \mathrm{mV}$. 0 . peak sodium current; $O$, residual current at the end of the depolarizing pulse: $\square$, amplitude of the slow tail current extrapolated to the moment of repolarization; $\triangle$, time constant of the slow tail current. Note that the vertical axis is in $\mathrm{nA}$ for $O .-$ and $\square$, but in $\mathrm{ms}$ for $\triangle$. Calibration: A, 2 ms and $2 \mathrm{nA} ; \mathrm{B}, 5 \mathrm{~ms}$ and $5 \mathrm{nA}$. 
ter and reached higher levels. The maximum increase in peak amplitude of the sodium current varied considerably between cells. Particularly in freshly differentiated cells a large increase was observed sometimes by more than $200 \%$ over control.

Other pyrethroids had qualitatively similar effects, but the extent to which the sodium current was prolonged differed markediy for the different pyretinroids. Phenothrin was the least effective pyrethroid (Fig. 2A). Fenfluthrin caused a marked slowing of the current during depolarization together with a large, slow sodium tail current after repolarization

A

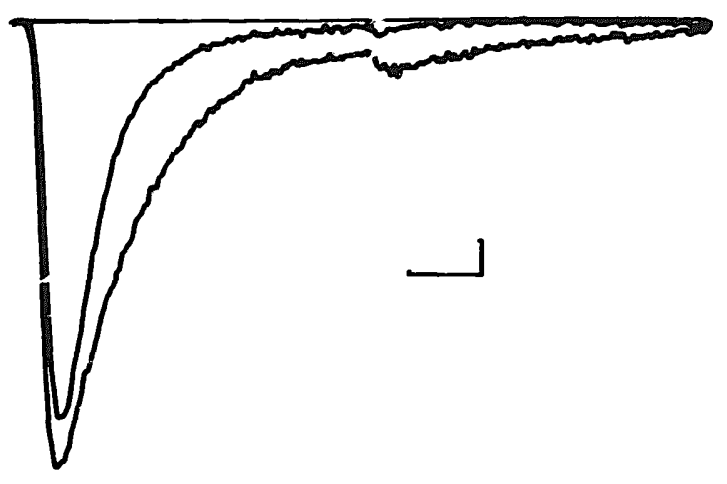

(Fig. 2B), while the effects of the $\alpha$-cyano pyrethroids cyphenothrin (Fig. 2C) and deltamethrin (Fig. 2D) were even more pronounced. The $\alpha$-cyano pyrethroids acted, however, much slower than phenothrin or fenfluthrin. Early after application of pyrethroid, or after low pyrethroid concentrations, two components could be distinguished in the declining phase of the sodium current during depolarization (Fig. 2B, C); a fast component similar to that of the normal sodium current and a much slower component. The contribution of the slow component to the total current increased with the duration of pyreth-
B

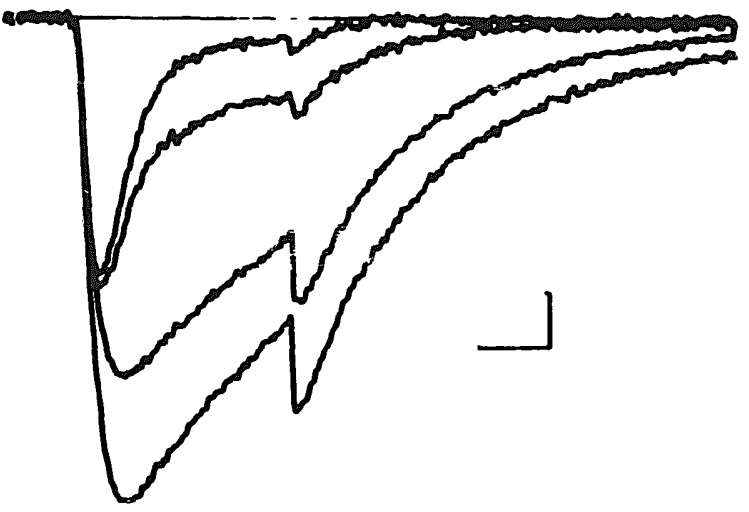

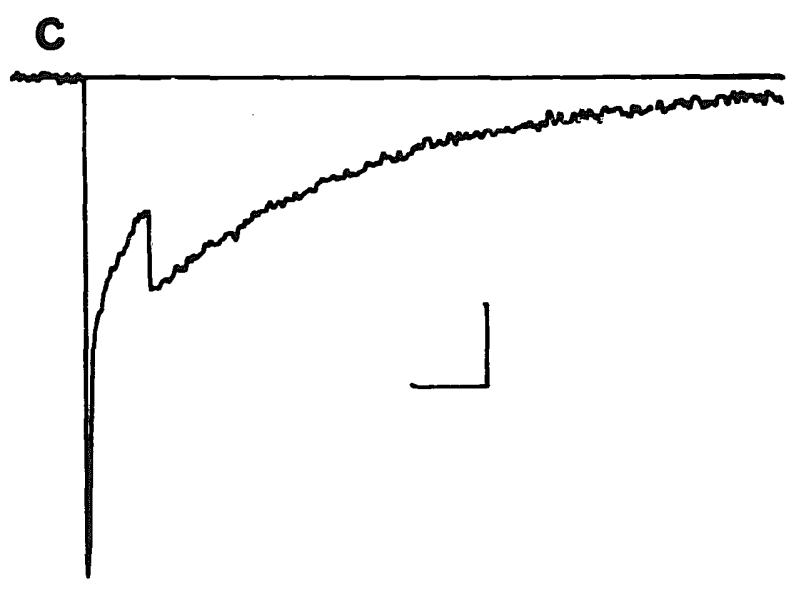

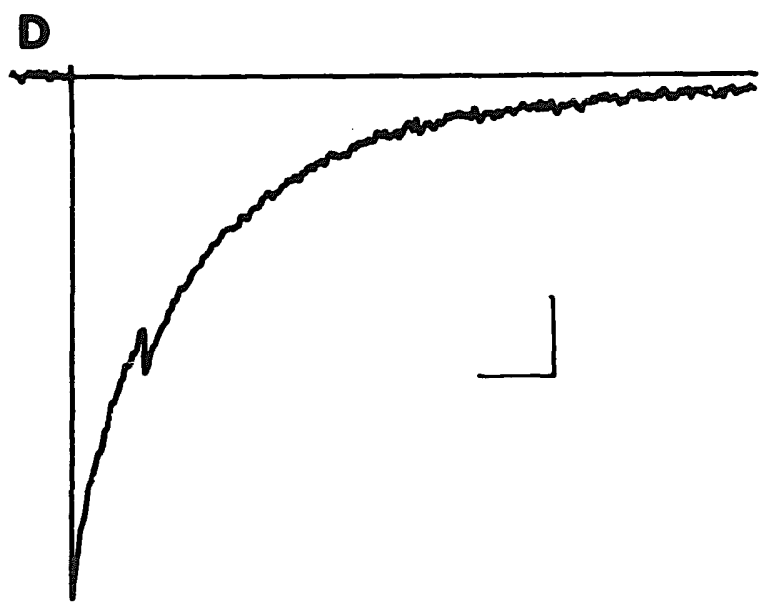

Fig. 2. Effect of different pyrethroids on the sodium current evoked by a depolarizing step to $-5 \mathrm{mV}$. A: sodium current during a $10 \mathrm{~ms}$ pulse before and after treatment with $100 \mu \mathrm{M}$ phenothrin for $20 \mathrm{~min}$. B: 4 superimposed traces of sodium current evoked by a $15 \mathrm{~ms}$ depolarizing pulse before and at $1.5,4.5$ and $30 \mathrm{~min}$ after application of $1 \mu \mathrm{M}$ fenfluthrin. Note the marked increase in peak amplitude during exposure to fenfluthrin. $C$ : sodium current during a $40 \mathrm{~ms}$ pulse after treatment with $25 \mu \mathrm{M}$ cyphenothrin. D: sodium current during a $200 \mathrm{~ms}$ pulse after treatment with $100 \mu \mathrm{M}$ deltamethrin. Calibration: $\mathrm{A}, 2 \mathrm{nA}$ and $2 \mathrm{~ms} ; \mathrm{B}, 2 \mathrm{nA}$ and $5 \mathrm{~ms} ; \mathrm{C}, 1 \mathrm{nA}$ and $50 \mathrm{~ms}$; $\mathrm{D}, 1 \mathrm{nA}$ and $200 \mathrm{~ms}$. 
roid exposure indicating that the number of affected sodium channels increased. The time course of decay of the slow comporients of the sodium current during depolarization and ufter repolarization of the membrane could be fitted by single exponentials. The respective time constants, $\tau_{\mathrm{s}}$ and $\tau_{\mathrm{t}}$, for the different pyrethroids, calculated after the effects had reached a steady state, are listed in Table I (the effects of pyrethroids on $\tau_{\mathrm{d}}$ are discussed below).

\section{Current-voltage (I-V) relationship}

Fig. 3A shows the I-V relationship before and after application of $1 \mu \mathrm{M}$ fenfluthrin. In control cells the sodium current activated between -35 and -25 $\mathrm{mV}$, reached a maxinum at -5 to $5 \mathrm{mV}$ and reversed sign between 40 and $50 \mathrm{mV}$. After fenfluthrin the sodium current activated at more negative potentials $(-45$ to $-55 \mathrm{mV})$ with a very slow time course of acti-

\section{TABLE I}

Time constants of the pyrethroid-induced slow component in the declining phase of the sodium current during depolarization to $-5 m V\left(t_{s}\right)$, of the slow sodium tail current after repolarization to $-95 \mathrm{mV}\left(\tau_{t}\right)$ and of the decline of slow tail current amplitude with increasing duration of a depolarizing step to $-5 \mathrm{mV}\left(\tau_{d}\right)$

\begin{tabular}{lcccc}
\hline & $\tau_{s}(m s)$ & & $\tau_{t}(m s)$ & $\tau_{d}(m s)$ \\
\hline Phenothrin & 3.0 & & 3.5 & 4.5 \\
Fenfluthrin & $12-35$ & & $15-30$ & $20-30$ \\
Cyphenothrin & $40-55$ & 140 & 55 \\
Deltamethrin & $125-160$ & $440-800$ & $140-155$ \\
\hline
\end{tabular}

vation. The maximum peak current increased markedly, while the maximum slope conductance was only slightly affected. Fenfluthrin did not affect the value of the reversal potential. The residual current had a similar voltage dependence as the peak so-

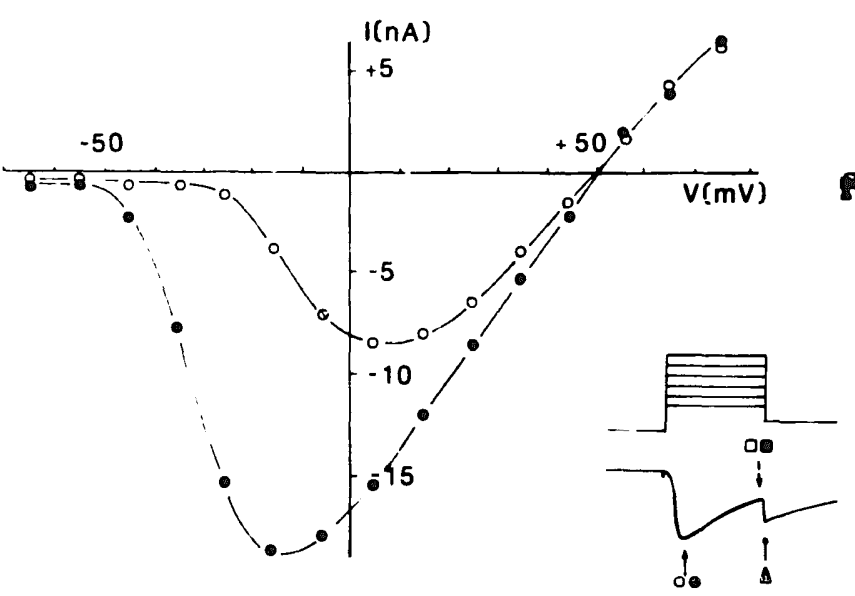

C

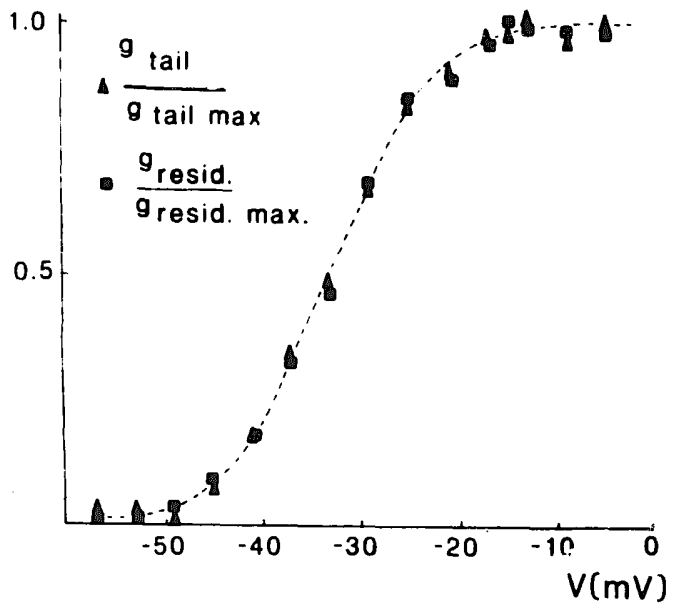

B

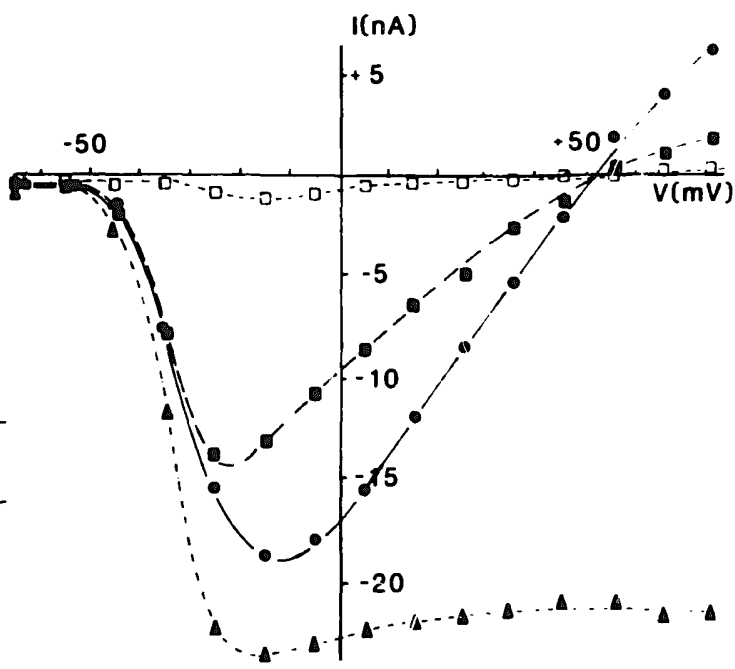

Fig. 3. A: current-voltage relationship of the peak sodium current before $(O)$ and after $(O)$ treatment with $1 \mu \mathrm{M}$ fenfluthrin for $85 \mathrm{~min}$. B: current-voltage relationship of the residual current before ( $\square$ ) and after (國) fenfluthrin. The voltage dependence of the peak sodium current is also depicted for comparison. Triangles represent the amplitude of the slow tail current extrapolated to the moment of repolarization (same cell as in A). C: relation between normalized tail conductance $(\Delta)$ as well as normalized conductance at the end of a depolarizing pulse ( and membrane potential during depolarization in a cell treated with $250 \mu \mathrm{M}$ deltamethrin for $15 \mathrm{~min}$. Inset shows pulse protocol. 
dium current and reversed sign at the same potential (Fig. 3B). The amplitude of the slow tail current increased in parallel with the activation of the sodium current during the depolarizing step and reached its maximum at the same potential as the residual current (Fig. 3B). With larger depolarizing steps the amplitude of the slow tail current decreased only slightly. The slow tail current was always directly proportional to the residual current, i.e. to the fraction of sodium channels which were open at the end of the depolarizing pulse, as illustrated in Fig. 3C. This figure shows how the normalized tail conductance and the normalized conductance at the end of the depolarizing pulse depend on the membrane potential during the depolarizing step.

\section{Sodium inactivation}

Steady-state sodium inactivation was investigated by conditioning the membrane for $200 \mathrm{~ms}$ at different potentials and by recording the peak sodium current during a subsequent test pulse of $15 \mathrm{~ms}$ to $-5 \mathrm{mV}$. Normalized peak currents were plotted against the potential of the conditioning prepulse (Fig. 4A). The iesults clearly show that $1 \mu \mathrm{M}$ fenfluthrin had no effect on steady-s+ate inactivation. Fig. 4A further shows the relation between the amplitude of the slow tail current and the prepulse potential. The two curves are identical which indicates that inactivated sodium channels do not contribute to the slow sodium tail current.

In control preparations the time constant of the de-
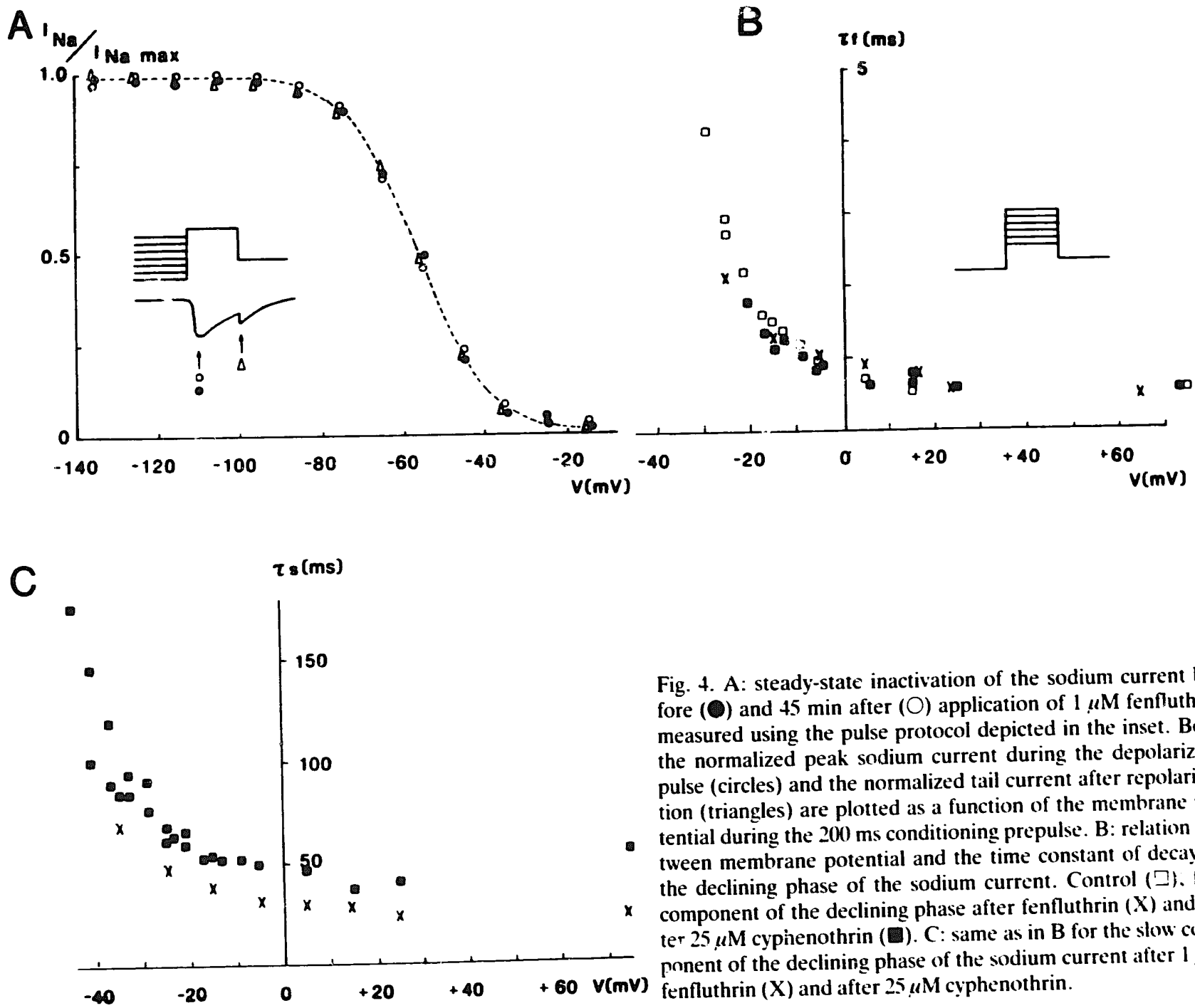

Fig. 4. A: steady-state inactivation of the sodium current hefore $(O)$ and $45 \mathrm{~min}$ after $(O)$ application of $1 \mu \mathrm{M}$ fenfluthrin measured using the pulse protocol depicted in the inset. Both the normalized peak sodium current during the depolarizing pulse (circles) and the normalized tail current after repolarization (triangles) are plotted as a function of the membrane po-

tential during the $200 \mathrm{~ms}$ conditioning prepulse. B: relation between membrane potential and the time constant of decay of the declining phase of the sodium current. Control ( $(2)$, fast

component of the declining phase after fenfluthrin $(X)$ and af ter $25 u \mathrm{M}$ cypinenothrin (娄). C: same as in B for the slow component of the declining phase of the sodium current after $1 \mu \mathrm{M}$ fenfluthrin $(\mathrm{X})$ and after $25 \mu \mathrm{M}$ cyphenothrin. 
clining phase of the sodium current $\left(\tau_{\mathrm{f}}\right)$ decreased steeply with increasing depolarizing steps in the negative membrane potential range (Fig. 4B). If a fast component was present in the declining phase of the sodium current after pyrethroid treatment, it had a time constant which did not differ significantly from $\tau_{\mathrm{f}}$ (Fig. 4B). The time constant of the pyrethroid-induced slow component $\left(\tau_{\mathrm{s}}\right)$ depended on pyrethroid structure (Table $\mathrm{I}$ ) and showed a similar voltage dependency as $\tau_{\mathrm{f}}$ (Fig. 4C).

The time course of recovery of sodium channels from inactivation was examined in order to determine whether the delayed closing of pyrethroid-affected channels during depolarization leads to a normal inactivated state. This was investigated by examining the time course of recovery of sodium channels from inactivation. The membrane was conditioned during $200 \mathrm{~ms}$ at $-145 \mathrm{mV}$ for removal of resting sodium inactivation. The membrane was then depolarized to $-5 \mathrm{mV}$ for $275 \mathrm{~ms}$ resulting in a maximum peak inward current and complete inactivation of the sodium current at the end of the pulse. After an interval of variable duration, in which the membrane potential was hyperpolarized to $-145 \mathrm{mV}$, a second depolarizing step was given and the normalized peak current was plotted against the duration of the interval. The time course of recovery from inactivation at $-145 \mathrm{mV}$ after $1 \mu \mathrm{M}$ fenfluthrin did not deviate markedly from the control as shown in Fig. 5. Similar results were obtained for deltamethrin.

\section{Slow sodium tail current}

Fig. 6A shows for a control recording how the fast sodium tail current, which was extrapolated to the moment of repolarization, depends on the duration of a preceding depolarizing step to $-5 \mathrm{mV}$. The time course of the normalized sodium conductance ciuring the depolarizing step is shown in the same figure. The two curves are almost identical which indicates that the fast sodium tail current is always proportional to the number of sodium channels which are open at the end of the depolarizing step. After pyrethroid treatment a slow component became apparent in the tail current. The fast coinponent of the tail current in pyrethroid-treated cells had the same time constant as the tail current in control cells. In the steady-state condition after administration of $1 \mu \mathrm{M}$ fenfluthrin the fast components of the tail current and of the de- clining phase of the depolarization-induced sodium current were both abolished. The slow tail current in these cells, measured after steps of increasirig duration, developed in parallel with the activation of the sodium current and reached its maximum at the peak of the sodium current (Fig. 6B). The decay of the tail current with longer depolarizing steps furthermore had the same time course as the sodium conductance during the step (Fig. 6B). Similar results were obtained for other pyrethroids, but the decline of the slow tail current amplitude with increasing duration of depolarization differed markedly for the different compounds (Fig. 6C). The curves in Fig. $6 \mathrm{C}$ could be fitted by a single exponential with a time constant $\tau_{\mathrm{d}}$ which was close to the time constant $\tau_{\mathrm{s}}$ of the slow component of the declining phase of the sodium current during depolarization (see Table I). These results clearly show that only sodium channels which are open at the end of the depolarizing step contribute to the slow tail current. The slow tail current in these cells developed in parallel with the activation of the sodium current and reached its maximum at the peak of the sodium current (Fig. 6B).

The rate of decay of the slow tail current did not only depend on pyrethroid structure, but depended also on the potential to which the membrane was repolarized as shown in Fig. 7A for fenfluthrin. The slow tail current showed a single exponential decay for the different pyrethroids with a time constant which increased steeply with membrane potential between -155 and $-85 \mathrm{mV}$ (Fig. 7B).

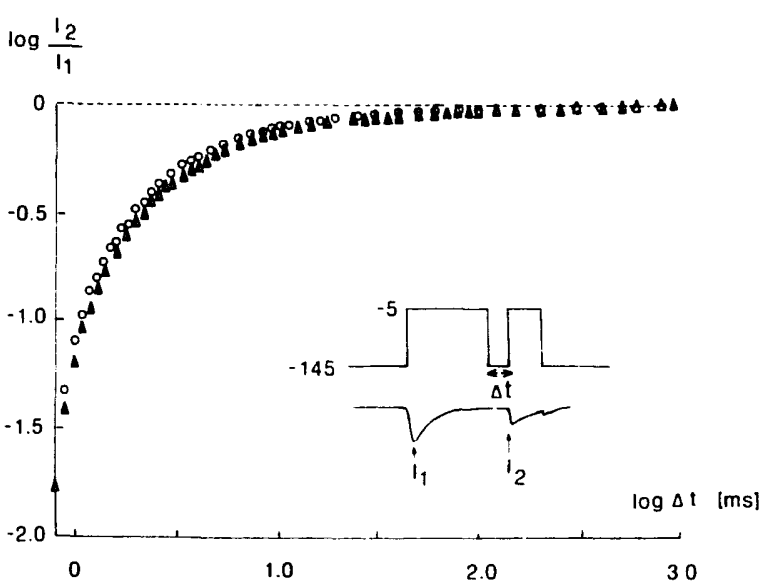

Fig. 5. Recovery from sodium inactivation before $(O)$ and after $(\Delta)$ treatment with $1 \mu \mathrm{M}$ fenfluthrin using the pulse protocol shown in the inset. For further details see text. Duration of the depolarizing pulses was 275 and $15 \mathrm{~ms}$, respectively. 
The instantaneous $\mathrm{I}-\mathrm{V}$ relationship for the normalized slow tail current at the moment of repolarization, as shown in Fig. 7C, was linear between -5 and $-65 \mathrm{mV}$ implying a constant conductance of the membrane for sodium ions within this range. However, if the membrane was repolarized to a potential below $-65 \mathrm{mV}$, the conductance started to decrease and a negative slope conductance was often observed below $-100 \mathrm{mV}$. When the membrane potential was varied $10 \mathrm{~ms}$ after the moment of repolarization a similar negative slope conductance was observed in the instantaneous I-V curve of cells treated with deltamethrin.

\section{Influence of temperature}

The influence of temperature on the rate of closing of sodium channels after pyrethroid treatment was

A
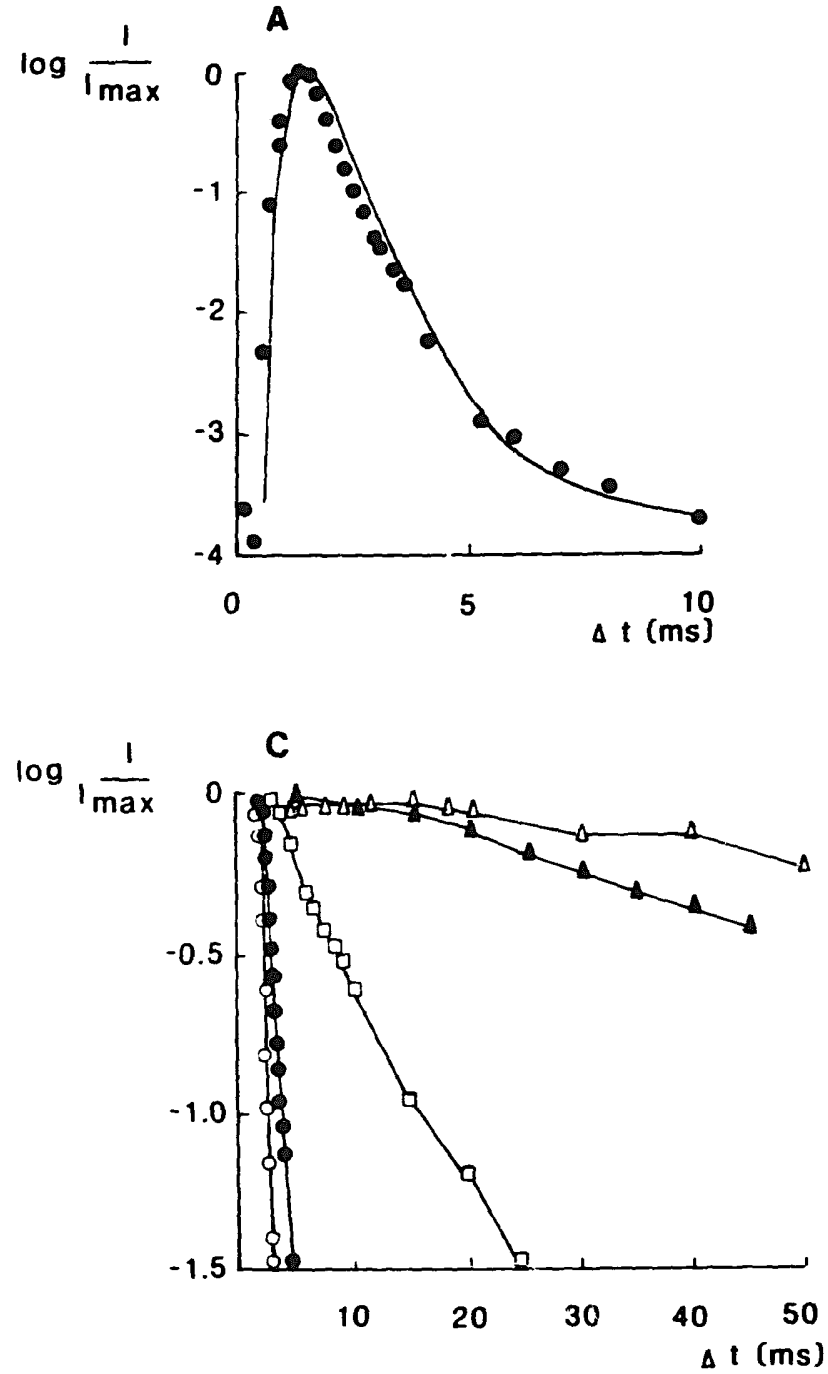

investigated in order to examine whether the temperature dependence of the gating of modified channels differed Srom that of normal channels. irrhenius plots of the rate of decay of the declining phase of the sodium current before and after application of $1 \mu \mathrm{M}$ fenfluthrin could be fitted by two straight lines with a break around $10^{\circ} \mathrm{C}$. Arrhenius plots for the rate of decay of the fast tail current in control cells and of the slow tail currents induced by different pyrethroids were linear over a large temperature range (Fig. 8). This suggests that during the tail current a single energy barrier limits the rate at which sodium channels close. The free energy of activation $\left(\Delta G_{a}\right)$ was calculated from the different Arrhenius plots ${ }^{36}$. The $\Delta G_{a}$ values for different cells treated with the same pyrethroid differed only slightly, whereas large differences were observed between cells in the values

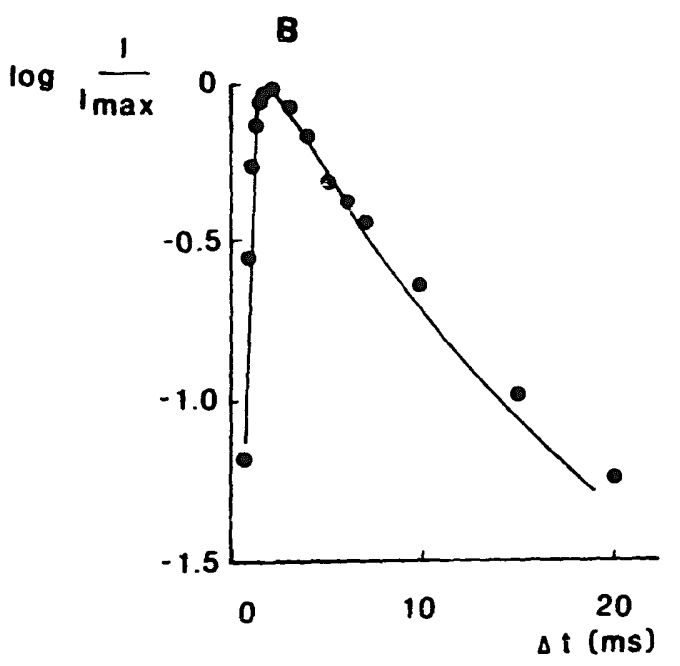

Fig. 6. Relation between normalized amplitude of the tail current (symbols) and duration of a depolarizing pulse to $-5 \mathrm{mV}$. A. control: B. after $1 \mu \mathrm{M}$ fenfluthrin. The continuous lines in $\mathrm{A}$ and $B$ represent the normalized sodium current during a single depolarizing pulse. In $C$ the declinc of the tail current amplitude with increasing duration of depolarization is shown for control $(O) .100 \mu \mathrm{M}$ phenothrin (O). $1 \mu \mathrm{M}$ fenfluthrin ( $\square$ ). 25 $\mu \mathrm{M}$ cyphenothrin $(\Delta)$ and $250 \mu \mathrm{M}$ deltamethrin $(\Delta)$. 


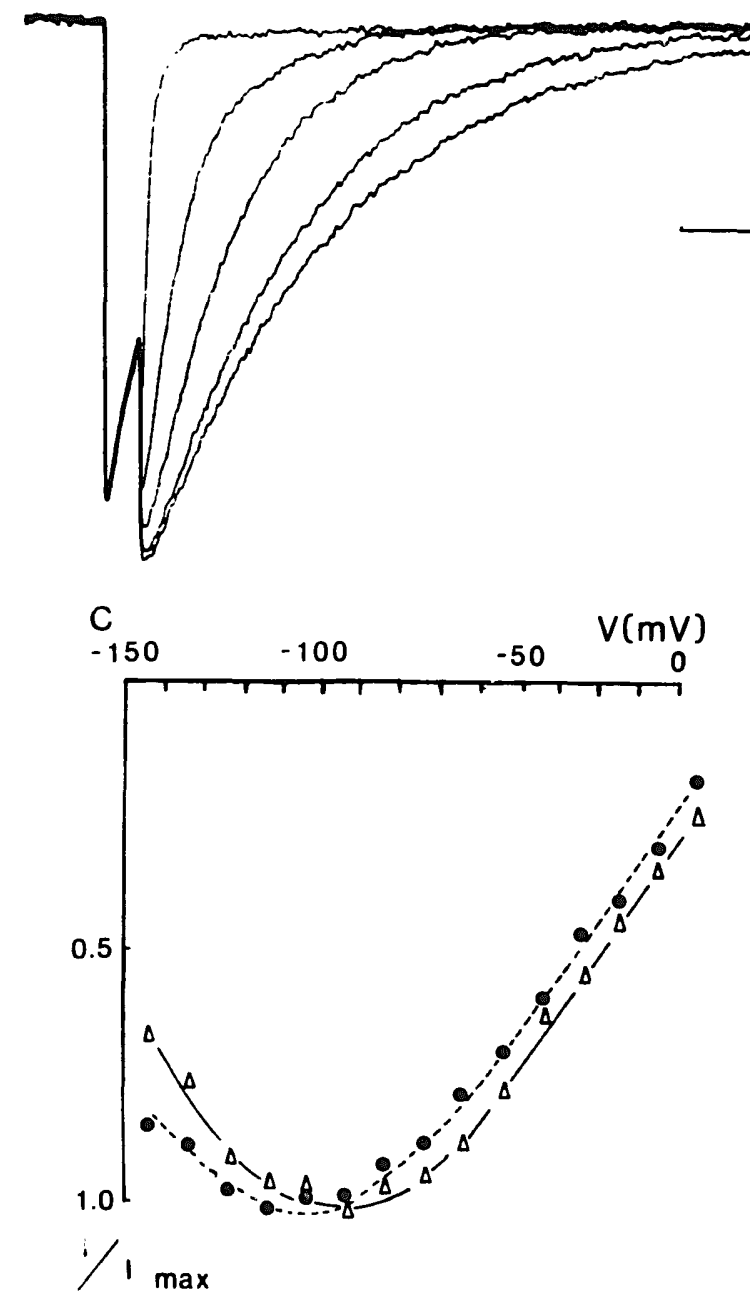




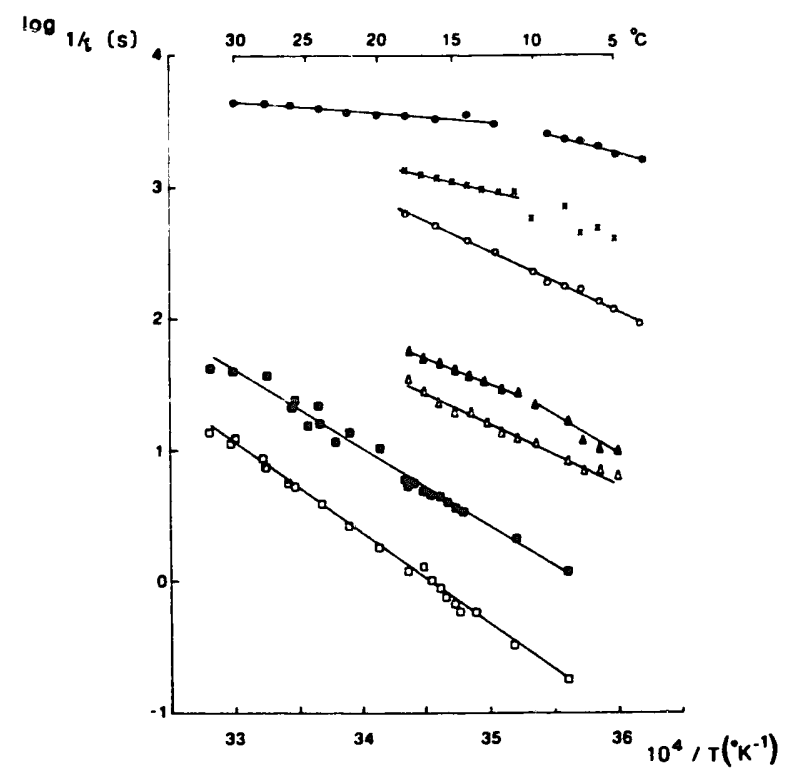

Fig. 8. Arrhenius plots for the rate of decay of the tail current after repolarization (open symbols) and for the rate of decay of the declining phase of the sodium current during depolarization (filled symbols). $\bigcirc$. control; $\triangle, 1 \mu \mathrm{M}$ fenfluthrin; $\square, 100 \mu \mathrm{M}$ deltamethrin. The Arrhenius plots for the rate of decay of the fast component in the declining phase of the sodium current after treatment with $1 \mu \mathrm{M}$ fenfluthrin is also shown (X). Straight lines represent least squares fit of the rate constants, which were averaged for each temperature.

constant of the pyrethroid-induced slow tail current (e.g. cyphenothrin vs phenothrin), there are large differences in the rate of decay of the tail current for $\alpha$-cyano pyrethroids (e.g. deltamethrin vs cyphenothrin) as well as for non-cyano pyrethroids (e.g. fenfluthrin vs phenothrin). This is in accordance with results from other studies in which a gradual transition was found between the effects of $\alpha$-cyano and non-cyano pyrethroids ${ }^{20.32}$. Two other non-cyano pyrethroids, cismethrin and permethrin also induced slow tail currents with a relatively rapid time constant

\section{TABLE II}

Values of the free energy of activation $\left(\Delta G_{a}\right)$ calculated from the Arrhenius plots of the rate of decay of the declining phase of the sodium current $\left(\Delta G_{l}\right)$ and of the rate of decay of the tail current $\left(\Delta G_{2}\right)$

\begin{tabular}{lll}
\hline & $\Delta G_{l}(\mathrm{~kJ} / \mathrm{mol})$ & $\Delta G_{2}(\mathrm{~kJ} / \mathrm{mol})$ \\
\hline Control & 62.5 & 64.5 \\
Fenfluthrin & 67.2 & 67.6 \\
Cyphenothrin & 68.1 & 69.2 \\
Deltamethrin & 69.6 & 71.0 \\
\hline
\end{tabular}

in neuroblastoma cells (unpublished). This moderate prolongation of the sodium current may be responsible for the absence of a detectable effect of several non-cyano pyrethroids on the action potential and on ${ }^{22} \mathrm{Na}^{+}$entry in neuroblastoma cells ${ }^{12}$.

The rate at which sodium channels close during the pyrethroid-induced slow tail current depends not oniy on pyrethroid structure, but also on the time of exposure, temperature and membrane potential. Arrhenius plots of the rate of decay of the tail current were linear, suggesting that a single energy barrier is involved in the closing of pyrcthroid-affected scdium channels after repolarization of the membrane. Like in the frog node of Ranvier the energy barrier after $a$-cyano pyrethroids $w_{i s} \rightarrow$ higher than after non-cyano pyrethroids ${ }^{36}$. The rate of decay of the slow tail current in the isolated frog node of Ranvier appeared not to be voltage dependent (unpublished). In crayfish and squid axon membranes, however, the time constant of the slow tail current decreased at hyperpolarized potentials ${ }^{17.18}$ in a similar way as observed in neuroblastoma cells.

The effects of pyrethroids on the sodium current during a depolarizing pulse clearly differ from those observed in other preparations. After pyrethroids the activation of sodium currents in neuroblastoma cells shifted to more negative potentials, which is in contrast with the frog node of Ranvier where no shift was observed $^{37}$. A marginal, but significant shift was described for the peak sodium current in squid axons treated with tetramsihrin ${ }^{17}$, whereas the activation of the pyrethroid-induced residual current in crayfish was shifted by $20 \mathrm{mV}$ in the hyperpolarizing direction $^{18}$. The delayed closing of sodium channels may be responsible for this shift in the voltage dependence of activation, as will be explained below. A mean open time of $0.5-1.5 \mathrm{~ms}$ has been reported for single sodium channels in neuroblastoma cells at room temperature ${ }^{1.24}$. The mean open time was independent of membrane potential over most of the activation range and did not depend on the latency of channel opening after the onset of a voltage step. The latency distribution of channel openings, however, depended strongly on membrane potential. Since the mean open time of sodium channels is prolonged by pyrethroids $^{39}$, the time course of activation of the sodium current after pyrethroids will therefore probably reflect the first latency distribution of openings of 
individual channels. The slow activation of the sodium current in the voltage range between -35 and -45 $\mathrm{mV}$ after treatment with pyrethroids is in keeping with results from single channel measurements showing that the first latencies for channel opening increase at negative potentials ${ }^{1}$. The results from single channel studies indicate that there is a relatively small probability that a substantial number of sodium channels will be simultaneously open during small depolarizing steps in normal preparations. After treatment with pyrethroids, however, sodium channels will stay open for a longer time than normal resulting in a detectable current at small depolarizations. This suggestion is strengthened by the fact that the activation of single channel currents is negatively shif $a d$ by $10-20 \mathrm{mV}$ relative to whole cell recordings in no. mal neuroblastoma cells ${ }^{24}$. A similar mechanism may underlie the observed increase in peak sodium current after pyrethroids, which was not found in frog or in invertebrate axonal membranes. An alternative explanation for the increased peak current is that the prolongation of single channel open times by pyrethroids enhances the probability of opening of neighboring channels. Such an interaction between neighboring sodium channels has been observed in neuroblastoma cells ${ }^{14}$. It is difficult to establish whether activation of 'silent' channels by pyrethroids may contribute to the increase in peak current, as has been postulated by Jacques et al. ${ }^{12}$. Since single channel recordings have shown that in neuroblastoma cells activation and inactivation are not independent ${ }^{1}$ extrapolation of the declining phase of the sodium current to the moment of depolarization is not a valid method for estimating the fraction of sodium channels available for activation. Therefore it is not possible to determine by analysis of the kinetics of the sodium current whether the increase in the peak sodium current after pyrethroids is due to an increase ir the number of available sodium channels or to an increase in mean open time of sodium channels ${ }^{5}$. The pyrethroid-induced increase in the peak current varied between cells and was especially large in cells which had been differentiated for only a few days. Thus in freshly differentiated cells a population of 'silent' sodium channels may exist, which may be activated by pyrethroid treatment.

The amplitude of the slow tail current in neuroblastoma cells increase $d$ in parallel with the rising phase of the sodium current during depolarization and reached its maximum when the membrane was repolarized at the peak of the sodium current. The rate of rise of the sodium current was not markedly affected by pyrethroids. The tail current was always proportional to the conductance at the end of the depolarizing pulse and no tail current was observed after depolarizing pulses leading to complete inactivation. However, in pyrethroid-treated frog nodes of Ranvier ${ }^{37}$ the sodium current during depolarization was affected to a minor extent only and pulses leading to almost complete inactivation were followed by large tail currents. It was therefore concluded that the tail current in the frog node of Ranvier is proportional to the number of sodium channels which had opened during depolarization ${ }^{37}$. Sodium inactivation in invertebrate axons was also slowed down by pyrethroid and like in neuroblastoma cells the slow tail current was always proportional to the fraction of pyrethroid-affected open channels at the end of the depolarizing pulse ${ }^{17.18}$. The large increase in the rate of decay of the tail current at membrane potentials below $-85 \mathrm{mV}$ indicates that the complex between the pyrethroid and the sodium channel is relatively unstable in this voltage range, possibly due " an increased force on the channel protein to change its conformation into a closed state. A similar mechanism may underlie the decrease of the time constant of slow inactivation at increasing voltage steps.

The prolongation of the open time of sodium channels makes it possible to study their conductance at potentials below $-50 \mathrm{mV}$. The instantaneous conductance at the end of a $15 \mathrm{~ms}$ depolarizing pulse in pyrethroid-treated cells was constant between -65 and $+5 \mathrm{mV}$, but decreased sharply below $-65 \mathrm{mV}$. A negative slope conductance was often observed in the voltage range between -155 and $-100 \mathrm{mV}$. Since tetramethrin did not significantly change the single channel conductance at a membrane potential around $-50 \mathrm{mV}$ in membrane patches of neuroblastoma cells ${ }^{39}$ it seems likely that the observed conductance decrease in the present experiments is not due to the treatment with pyrethroids. A similar rectification of tail current instantane Jus I-V curves at potentials more negative than $-40 \mathrm{mV}$ was also observed under control conditions in Myxicola ${ }^{7}$ and squid giant axons ${ }^{40}$. This rectification increases with increasing extracellular calcium concentration, ap- 
pears not to be due to a change in single channel kinetics and is not affected by the pyrethroid tetramethrin $^{39,40}$.

On the basis of the present results the interaction between pyrethroids and sodium channels in neuroblastoma cells can be described in the following manner. Whenever sodium channels pass through an open state, conformational changes involved in the closing of open channels are impeded by pyrethroids, whereas conformational changes involved in the opening of sodium channels or in the removai of sodium channel inactivation are not affected. Two different models of interaction of pyrethroids with sodium channels are compatible with these results. Pyrethroids may rapidly associate with the sodium channel protein in its open conformation, delay the transition to the closed state and dissociate rapidly from their binding site when the protein returns to a closed conformation. On the other hand, pyrethroids may be bound to the channel protein in such a way that only conformational changes from the open to the closed state are impeded. The second alternative appears

\section{REFERENCES}

1 Aldrich, R.W., Corey, D.P. and Stevens, C.F., A reinterpretation of mammalian sodium channel gating based on single channel recording, Nature (London), 306 (1983) 436-441.

2 Amano, T., Richelson, E. and Nirenberg, P.G., Neurotransmitter synthesis by neuroblastoma clones, Proc. Natl. Acad. Sci. U.S.A., 69 (1972) 258-263.

3 Brown, K.M. and Dennis, J.E., Derivative free analogues of the Levenberg-Marquardt and Gauss algorithms for nonlinear least squares approximations, Numerische Mathematik, 18 (1972) 289-297.

4 Catterall, W.A., Studies of voltage-sensitive sodium channels in cultured cells using ion-flux and ligand-binding methods. In P.G. Nelson and M. Lieberman (Eds.), Excitable Cells in Tissue Culture, Plenum, New York, 1981, pp. 279-314.

5 French, R.J. and Horn, R., Sodium channel gating: models, mimics and modifiers, Annu. Rev. Biophys. Bioeng., 12 (1983) 319-356.

6 Ghiasuddin, M. and Soderlund, D.M., Pyrethroid insecticides: potent, stereospecific enhancers of mouse brain sodium channel activation, Pestic. Biochem. Physiol., 24 (1985) 200-206.

7 Goldman, L. and Hahin, R., Initial conditions and the kinetics of the sodium conductance in Myxicola giant axons. II. Relaxation experiments, J. Gen. Physiol., 72 (1978) 879-898.

8 Grishchenko, I.I., Naumov, A.P. and Zubov. A.N.. Gating and selectivity of aconitine-modiffed sodiunt channels in neuroblastoma cells, Neuroscience, 9 (1983) 549-554. most likely, since pretreatment of neuroblastoma cells with inactive pyrethroids prevents the development of a depolarizing afterpotential after active pyrethroids $^{12}$. The relatively slow time course of development of pyrethroid effects after exposure of neuroblastoma cells also suggests that pyrethroids do not interact rapidly with the mammalian sodium channel protein.

\section{ACKNOWLEDGEMENTS}

We thank Dr. Naumann and Bayer AG, Rousell UCLAF and Shell Research Ltd. for the gift of pyrethroids. Neuroblastoma cells were obtained through Peter Meyer of the Hubrecht Laboratory and were cultured by Ine van Heugten-Hassing. We thank Dr. Fred Quandt for sharing his experience with the suction electrode, voltage clamp technique. This work was supported by the Foundation for Medical Research FUNGO/ZWO and by Shell Internationale Research Maatschappij.

9 Grishchenko, I.I., Zubov, A.N. and Naumov, A.P., Aconitine modification of sodium channels in the neuroblastoma cell membrane, Tsitologija, 24 (1982) 658-667.

10 Hodgkin, A.L. and Huxley, A.F., A quantitative description of membrane current and its application to conduction and excitation in nerve, J. Physiol. (London), 117 (1952! 500-544.

11 Huang, L.M., Moran, N. and Ehrenstein, G., Batrachotoxin modifies the gating kinetics of sodium channels in internally perfused neuroblastoma cells, Proc. Natl. Acad. Sci. U.S.A., 79 (1982) 2082-2085.

12 Jacques, Y., Romey, G., Cavey, M.T., Kartalovski, B. and Lazdunski, M., Interaction of pyrethroids with the $\mathrm{Na}^{+}$ channel in mammalian neuronal cells in culture, Biochim. Biophys. Acta, 600 (1980) 882-897.

13 Omitted.

14 Kiss, T. and Nagy, K., Interaction between sodium channels in mouse neuroblastoma cells, Eur. Biophys. J., 12 (1985) 13-18.

15 Lazdunski, M., Barhanin, J., Frelin, C., Hugues, M. and Romey, G., Ion channels in the control of states of excitability in neuronal membranes. In Basic Mechanisms of Neuronal Hyperexcitability, Alan R. Liss, New York, 1983.

16 Lee, K.S., Akaike, N. and Brown, A.M.. The suction pipette method for internal perfusion and voltage clamp of small excitable cells, J. Neurosci. Meth., 2 (1980) 51-78.

17 Lund, A.E. and Narahashi, T., Kinetics of sodium channel modification by the insecticide tetramethrin in squid axon membranes, J. Pharmacol. Exp. Ther.. 219 (1981) 464-473.

18 Lund, A.E. and Narahashi, i.., Modification of sodium channel kinetics by the insecticide tetramethrin in crayfish 
giant axons, Neurotox., 2 (1981) 213-229.

19 Lund, A.E. and Narahashi, T., Dose-dependent interaction of pyrethroid isomers with sodium channels of squid axon membranes, Neurotox., 3 (1982) 11-24.

20 Lund, A.E. and Narahashi, T., Kinetics of sodium channel modification as the basis for the variation in the nerve membrane effects of pyrethroids and DDT analogs, Pestic. Biochem. Physiol., 20 (1983) 203-216.

21 Moolenaar, W.H. and Spector, I., Ionic currents in cultured mouse neuroblastoma cells under voltage-clamp conditions, J. Physiol. (London), 278 (1978) 265-286.

22 Moolenaar, W.H. and Spector, I., The calcium action potential and a prolonged calcium dependent after-hyperpolarization in mouse neuroblastoma cells, J. Physiol. (London), 292 (1979) 297-306.

23 Moolenaar, W.H. and Spector, I., The calcium current and the activation of a slow potassium conductance in voltageclamped mouse neuroblastoma cells, J. Physiol. (London), 292 (1979) 307-323.

24 Nagy, K., Kiss, T. and Hof, D., Single Na channels in mouse neuroblastoma cell membrane; indications for two open states, Pflügers Arch., 399 (1983) 302-308.

25 Narahashi, T., Effects of insecticides on excitable tissues. In J.W.L. Beament, J.E. Treherne and V.B. Wigglesworth (Eds.), Advances in Insect Physiology, Vol. 8, Academic, New York, 1971, pp. 1-93.

26 Narahashi, T., Effects of insecticides on riervous conduction and synaptic transmission. In C.F. Wilkinson (Ed.), Insecticide Biochemistry and Physiology, Plenum, New York, 1976, pp. 327-352.

27 Naumann, K., Chemie der Synthetischen Pyrethroid-Insektizide. Chemie der Pflanzenschütz- und Schädlingsbekämpfungsmittel, Band 7, Springer, Berlin, 1981.

28 Nonner, W., Relations between the inactivations of sodium channels and the immobilization of gating charge in frog myelinated nerve, J. Physiol. (London), 299 (1980) 573-603.

29 Quandt, F.N. and Narahashi, T., Modifications of single $\mathrm{Na}^{+}$channels by batrachotoxin, Proc. Natl. Acad. Sci. U.S.A., 79 (1982) 6732-6736.
30 Roche, M., Frelin, C., Bruneau, P. and Meinard, C., Interaction of tralomethrin, tralocythin, and related pyrethroids in $\mathrm{Na}^{+}$channels of insect and mammalian neuronal cells, Pestic. Biochem. Physiol., 24 (1985) 306-316.

31 Ruigt, G.S.F., Pyrethroids. In G.A. Kerkut and L.I. Gilbert (Eds.), Comprehensive Insect Physiology, Biochemistry und Pharmainlogy. "ol. 12, Pergamon, Oxford, 1985, pp. 183-261.

32 Ruigt, G.S.F. and Van den Bercken, J., Action of pyrethroids on a nerve-muscle preparation of the clawed frog, Xenopus laevis, Pestic. Biochem. Physiol., 25 (1986) 176-187.

33 Ruigt, G.S.F., Klis, J.F.L. and Van den Bercken, J., Pronounced repetitive activity induced by the pyrethroid insecticide, fenfluthrin, in the slowly adapting stretch receptor neuron of the crayfish, J. Comp. Physiol., 159 (1986) 43-53.

34 Spector, I., Electrophysiology of clonal nerve cell lines. In P.G. Nelson and M. Lieberman (Eds.), Excitable Cells in Tissue Culture, Plenum, New York, 1981, pp. 3:7-274.

35 Vijverberg, H.P.M. and Van den Bercken, J., Action of pyrethroid insecticides on the vertebrate nervous system, Neuropathol. Appl. Neurobiol., 8 (1982) 421-440.

36 Vijverberg, H.P.M., Van der Zalm, J.M., Van Kleef, R.G.D.M. and Van den Bercken, J., Temperature- and structure-dependent interaction of pyrethroids with the sodium channels in frog node of Ranvier, Biochim. Biophys. Acta, 728 (1983) 73-82.

37 Vijverberg, H.P.M., Van der Zalm, J.M. and Van den Bercken, J., Similar mode of action of pyrethroids and DDT on sodium channel gating in myelinated nerves, $\mathrm{Na}$ ture (London), 295 (1982) 601-603.

38 Wouters, M. and Van den Bercken, J., Action of pyrethroids, Gen. Pharmacol., 9 (1978) 387-398.

39 Yamamoto, D., Yeh, J.Z. and Narahashi, T., Voltage-dependent calcium block of normal and tetramethrin modified single sodium channels, Riophys. J., 45 (1984) 337-344.

40 Yamamoto, Y., Yeh, J.Z. and Narahashi, T., Ion permeation and selectivity of squid axon sodium channels modified by tetramethrin, Brain Rescarch, 372 (1986) 193-197. 